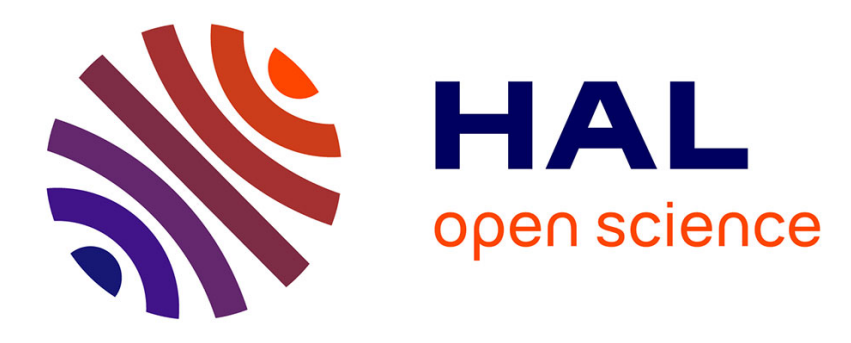

\title{
Adaptive Step-size Iterative Equalization for Underwater Communication
}

Zi Ye, Benoit Geller, Tarak Arbi

\section{To cite this version:}

Zi Ye, Benoit Geller, Tarak Arbi. Adaptive Step-size Iterative Equalization for Underwater Communication. IEEE Oceans 21, Sep 2021, San Diego, United States. hal-03426901

\section{HAL Id: hal-03426901 https://hal.science/hal-03426901}

Submitted on 12 Nov 2021

HAL is a multi-disciplinary open access archive for the deposit and dissemination of scientific research documents, whether they are published or not. The documents may come from teaching and research institutions in France or abroad, or from public or private research centers.
L'archive ouverte pluridisciplinaire HAL, est destinée au dépôt et à la diffusion de documents scientifiques de niveau recherche, publiés ou non, émanant des établissements d'enseignement et de recherche français ou étrangers, des laboratoires publics ou privés. 


\section{Adaptive Step-size Iterative Equalization for Underwater Communication}

\author{
$1^{\text {st }} \mathrm{Zi} \mathrm{Ye}$ \\ Department U2IS, ENSTA Paris \\ Institut Polytechnique de Paris, France \\ zi.ye@ensta-paris.fr
}

\author{
$2^{\text {nd }}$ Tarak Arbi \\ Department U2IS, ENSTA Paris \\ Institut Polytechnique de Paris, France \\ tarak.arbi@ensta-paris.fr
}

\author{
$3^{\text {rd }}$ Benoit Geller \\ Department U2IS, ENSTA Paris \\ Institut Polytechnique de Paris, France \\ benoit.geller@ensta-paris.fr
}

\begin{abstract}
This paper presents a fully adaptive Minimum Mean Square Error (MMSE) iterative equalization with a joint phase estimation, using various adaptive step-sizes scheme. To meet the requirement of fast convergence and low MSE over time-varying channels, we propose an original self-optimized algorithm whose step-sizes are updated adaptively and assisted by soft-information provided by the channel decoder in an iterative manner. Simulation results show that our proposal achieves better performance over various multipath time-varying channels, compared to the conventional equalizer using a fixed step-size.
\end{abstract}

Index Terms-turbo equalization, phase estimation, adaptive algorithms

\section{INTRODUCTION}

The choice of step-sizes for adaptive algorithms has a considerable impact, in particular on the UnderWater Acoustic (UWA) communication system performance; however, by essence, this choice is difficult in practice and often leads to severe performance degradation.

We consider this issue for two adaptive algorithms that are crucial at the receiving side when the channel is doubly selective: equalization and phase synchronisation. Indeed, iterative turbo equalization [1]-[3] can be difficult when the InterSymbol Interference (ISI) due to multipath propagation is severe; also, accurate adaptive phase recovery may be critical in the case of high Doppler frequency variations [4]-[10].

The performance of the standard Minimum Mean Square Error (MMSE) iterative equalization depends on its constant step-size [3], [11]-[14]; in particular, the step-size controls both the convergence speed and the steady-state MSE of the algorithm; hence it affects significantly the performance of the adaptive equalizer. Furthermore, for time-varying channels such as underwater acoustic channels, algorithms using constant step-sizes are unable to achieve a good trade-off between the performance and the convergence speed. However, most of the existing literature concerned with MMSE adaptive step-size filters [8], [9], [15]-[17] did not consider the case of turbo equalization; [18] (resp. [19]) proposed an iterative equalization scheme based on the least-symbol-error-rate [20] with a constant (resp. variable) step-size; besides it was not designed to track phase drifts of challenging channels, its high complexity makes it poorly suited for both embedded systems and fast time-varying channels.

In this paper, we propose an original adaptive step-size algorithm for joint equalization and phase estimation for

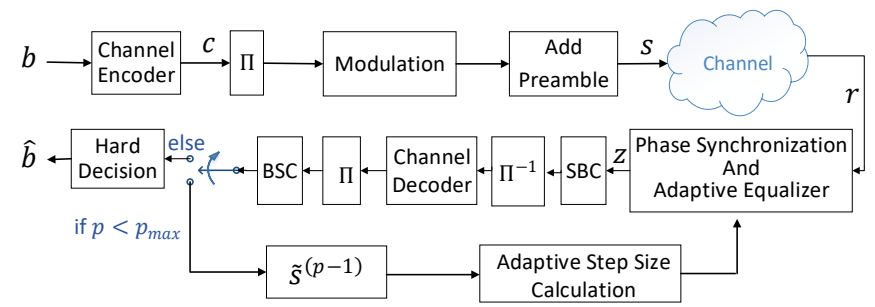

Fig. 1. System model.

which the soft information exchanged between the equalizer and the channel decoder can also be used for adjusting the adaptive step-size, as it is an appealing indicator of the symbol reliability. The proposed algorithm for joint phase recovering and equalization allows a faster convergence speed, a better tracking performance over time-varying channel and a lower mean square error compared to the constant step-size case.

The paper is organized as follows. Section II describes the system model. Section III presents the adaptive step-size equalization - phase estimation algorithm. Numerical results obtained over some time-varying channels and a real UWA channel are displayed in Section IV. At last, some conclusions are made in Section V.

\section{SYSTEM MODEL}

The transmission scheme is displayed in Fig. 1. A binary information sequence $\boldsymbol{b}$ is encoded by a channel encoder into a sequence of coded symbols $c$ [21], [22]. A bit interleaver $\Pi$ is then performed to increase the code diversity, and each set of $m=\log _{2} M$ interleaved coded bits are mapped into a M-QAM complex symbol $s_{k}$ in the alphabet $\chi$. A known training sequence is added to the coded symbols, which allows the receiver to perform several baseband processing such as synchronization and frame detection [2], [23]-[27]. The obtained signal $s$ is finally transmitted through a doublyselective channel. In our simulations, we tested our proposal over an underwater channel provided by [28] (e.g. Fig. 2 shows some impulse responses of a $\mathrm{BCH} 1$ channel measured off the commercial harbour of Brest in France, over a distance of 800 $\mathrm{m}$ at different times) and also a synthetic channel provided by 


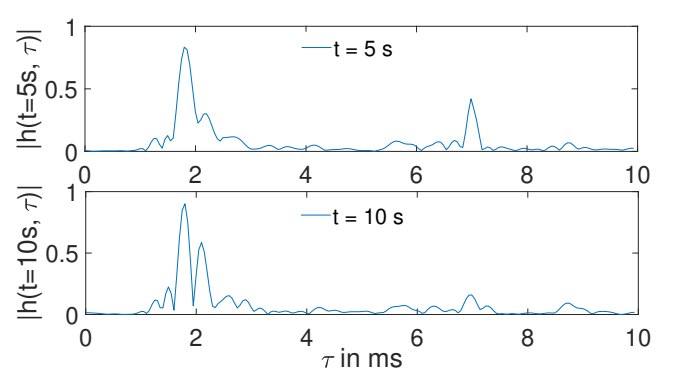

Fig. 2. Impulse responses at time $\mathrm{t}=5,10 \mathrm{~s}$ of $\mathrm{BCH} 1$ channel.

[2]. The received symbols can be written as:

$$
r_{k}=\sum_{l} h_{l}(k) s_{k-l}+w_{k},
$$

where at time index $k, h_{l}(k)$ indicates the channel response, and $w_{k}$ is an Additive White Gaussian Noise (AWGN).

At the receiver side of Figure 1, both the phase variation and ISI caused by the time-varying multipath channel need to be combated to achieve good performance through several signal processing algorithms. Therefore, the receiver performs the equalization and phase synchronization algorithm as described in the next section. Thereafter, the obtained symbols $z_{k}$ are fed into the Symbol to Bit Converter (SBC) in order to compute the Log-Likelihood Ratio (LLR) values from the equalized symbol $z_{k}$ [2]:

$$
z_{k}=\beta_{0} \tilde{s}_{k}+\nu_{k},
$$

where $\beta_{0} \tilde{s}_{k}$ is the desired signal with a constant bias factor $\beta_{0}$ and $\nu_{k}$ is a Gaussian noise of variance $\sigma_{v}^{2}$. The LLRs of the mapped bits $l^{i}(s),\left(i=0,1, \ldots, \log _{2} M-1\right)$ is given by:

$$
L L R\left(l^{i}(s)\right)=\ln \frac{\sum_{x \in \boldsymbol{\chi}_{b}^{1}} \exp \left(-\frac{\left|z_{k}-\beta_{0} x\right|^{2}}{\sigma_{v}^{2}}\right)}{\sum_{x \in \boldsymbol{\chi}_{b}^{0}} \exp \left(-\frac{\left|z_{k}-\beta_{0} x\right|^{2}}{\sigma_{v}^{2}}\right)},
$$

where $l^{i}(s)$ is the $i$-th bit of symbol $s$ and $\chi_{b}^{i}$ denotes the set of the constellation points whose $i$-th bit is $b=1$ or 0 .

Then, the channel decoder is applied; its extrinsic soft information output is used by the Bit to Symbol Converter (BSC) to obtain the soft estimated symbol $\bar{s}_{k}$ [2]. Finally, the symbols $\bar{s}_{k}$ are used in the next iteration of our proposed joint equalization - phase synchronization algorithm. Finally, after $p_{\max }$ iterations (i.e. equalization/channel decoder), a hard decision on the LLRs at the output of the decoder is made to estimate the user information bits.

\section{THE PROPOSED ADAPTIVE TURBO EQUALIZER}

\section{A. Equalizer structure}

Fig. 3 presents the equalizer - phase estimator structure; two filters $\boldsymbol{f}_{k}$ and $\boldsymbol{g}_{k}$ are fed by the output channel sequence $\boldsymbol{R}_{k}=\left[r_{k+L_{1}}, \cdots, r_{k-L_{1}}\right]^{T}$ and the symbol sequence $\tilde{\boldsymbol{S}}_{k}=$ $\left[\tilde{s}_{k+L_{2}}, \cdots, \tilde{s}_{k-L_{2}}\right]^{T}$ respectively, where $\tilde{s}_{k}$ denotes either the

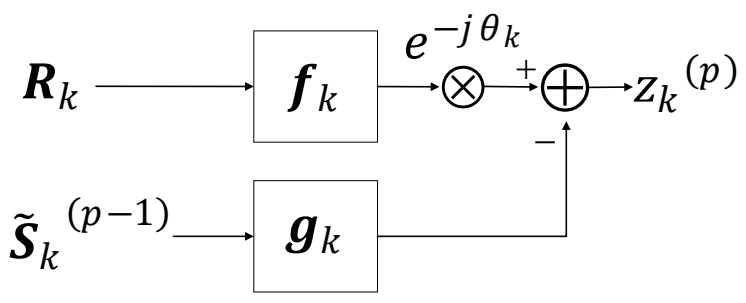

Fig. 3. Joint equalizer - phase estimator structure.

known preamble or the soft estimated symbol at the previous iteration for $p>0$. The equalized symbol at time $k$ is:

$$
z_{k}=\boldsymbol{f}_{k}^{T} \boldsymbol{R}_{k} e^{-j \theta_{k}}-\boldsymbol{g}_{k}^{T} \tilde{\boldsymbol{S}}_{k},
$$

where $\boldsymbol{f}_{k}=\left[f_{-L_{1}}(k), \cdots, f_{L_{1}}(k)\right]^{T} \quad$ (resp. $\boldsymbol{g}_{k}=$ $\left.\left[g_{-L_{2}}(k), \cdots, g_{0}(k)=0, \cdots, g_{L_{2}}(k)\right]^{T}\right)$ is a transversal filter equalizer of length $2 L_{1}+1$ (resp. $2 L_{2}+1$ ), and $\theta_{k}$ is the estimated phase error; at each iteration $p$ (less than $p_{\max }$ ) the transversal filter $\boldsymbol{g}_{k}$ aims at filtering the residual ISI on the equalized symbol at the previous iteration. The index $(p)$ is dropped in the sequel.

The coefficients of the filters are updated in order to minimize the MSE given by:

$$
J\left(\boldsymbol{f}_{k}, \boldsymbol{g}_{k}, \theta_{k}\right)==\underset{k}{\mathbb{E}}\left(\underset{x}{\mathbb{E}}\left(\operatorname{Pr}\left(x \mid z_{k}\right)\left|x-z_{k}\right|^{2}\right)\right),
$$

where $\mathbb{E}(d)$ designates the expectation of $d$ and:

$$
\operatorname{Pr}\left(x \mid z_{k}\right)=\frac{\operatorname{Pr}(x) \operatorname{Pr}\left(z_{k} \mid x\right)}{\sum_{x^{\prime} \in \chi} \operatorname{Pr}\left(x^{\prime}\right) \operatorname{Pr}\left(z_{k} \mid x^{\prime}\right)},
$$

with:

$$
\operatorname{Pr}\left(z_{k} \mid \tilde{x}\right)=\frac{1}{\pi \sigma_{v}^{2}} \exp \left(-\frac{\left|z_{k}-\beta_{0} \tilde{x}\right|^{2}}{\sigma_{v}^{2}}\right) .
$$

It is important to note that in contrast to previous works [2], [8], [9], [11]-[13], the calculation of the MSE of equation (5) encompasses all synchronisation modes (Data-Aided (DA), Non-Data-Aided (NDA) and Code-Aided(CA)); in particular, for the CA mode, the probability of the constellation point $x \in \chi$ can be computed using the LLR values (see (3)) at the output of the channel decoder.

With some basic mathematical derivations, we can obtain $\frac{\partial J}{\partial \boldsymbol{f}_{k}}, \frac{\partial J}{\partial \boldsymbol{g}_{k}}$ and $\frac{\partial J}{\partial \theta_{k}}$.

Using the gradient descent algorithm, the filter coefficients $\boldsymbol{f}_{k}$, $\boldsymbol{g}_{k}$ and the estimated phase error $\theta_{k}$ are updated as:

$$
\left\{\begin{array}{l}
\boldsymbol{f}_{k+1}=\boldsymbol{f}_{k}-\frac{\mu}{2} \frac{\partial J}{\partial \boldsymbol{f}_{k}} \\
\boldsymbol{g}_{k+1}=\boldsymbol{g}_{k}-\frac{\mu}{2} \frac{\partial J}{\partial \boldsymbol{g}_{k}} \\
\theta_{k+1}=\theta_{k}-\frac{\gamma}{2} \frac{\partial J}{\partial \theta_{k}}
\end{array}\right.
$$

where $\mu$ and $\gamma$ are the step-sizes. 


\section{B. Adaptive step-size algorithm}

We propose to optimize the step-sizes $\mu$ and $\gamma$ in an adaptive manner on the coefficients trajectory so as to minimize the MSE (see (5)). Using the gradient descent algorithm, the stepsizes $\mu$ and $\gamma$ are updated as:

$$
\left\{\begin{array}{l}
\mu_{k+1}=\mu_{k}-\frac{\alpha}{2} \frac{\partial J}{\partial \mu} . \\
\gamma_{k+1}=\gamma_{k}-\frac{\beta}{2} \frac{\partial J}{\partial \gamma} .
\end{array}\right.
$$

where $\frac{\partial J}{\partial \mu}$ and $\frac{\partial J}{\partial \gamma}$ can easily be obtained with some basic mathematical derivations.

It should be noted that, in contrast to state-of-the-art solutions [2], the proposed adaptive step-sizes are continuously and blindly updated on the whole information burst and exploit the soft information of each received symbol provided by the channel decoder at the previous iteration; this contributes to a good equalization - phase error estimation performance. In addition, note that for the adaptive step-size equalizer, the values of the second-order step-sizes $\alpha$ and $\beta$ do not influence considerably the system performance, and can therefore be chosen in a large range.

\section{SIMULATION RESULTS}

\section{A. Synthetic time varying channel}

In our simulations, a training sequence of 100 symbols is transmitted before each user frame. At the receiver side, $p_{\max }$ is fixed to 10 . We consider the synthetic channel [2]:

$$
h_{l}(k)=\sqrt{\frac{P_{l}}{I}} \sum_{i=1}^{I} e^{j\left(k \epsilon \pi \cos \psi_{l, i}+\zeta_{l, i}\right)},
$$

where the Doppler shift is characterised by $\epsilon, P_{l}$ denotes the mean power of the $l$-th channel path, $\psi_{l, i}$ and $\zeta_{l, i}$ are uniform random variables over $[0,2 \pi[; I$ is fixed to 10 and (10) has three paths having all the same mean power [2] in our simulation.

We evaluate the performance of our proposal by considering 2 scenarios, for all of which the initial Doppler shift is equal to 0.001 and linearly changes to reach the value $\epsilon_{\max }$ at the end of the received block. In the first scenario, we consider a relatively slow variation where $\epsilon_{\max }^{1}=0.002$, for the second scenario, the Doppler shift at the end of the received block is equal to $\epsilon_{\max }^{2}=0.005$. The number of taps for $\boldsymbol{f}_{k}$ and $\boldsymbol{g}_{k}$ are equal to 13 and 19 respectively, the central coefficient of $\boldsymbol{f}_{k}$ is set to 1 .

Fig. 4 (resp. Fig. 5) compares the BER performance between the proposed fully adaptive step-size algorithm over the whole frame, the so-called "CS tracking" algorithm for which the step size is adaptive during the initial training sequence and then fixed during the information frame to the value reached at the end of the preamble, and finally the algorithm for which the step size is fixed to the initial value for all symbols transmitted over the time varying channel (10) with $\epsilon_{\max }^{1}$ (resp. $\epsilon_{\max }^{2}$ ) for both the QPSK and 16-QAM constellations. It can first be observed that for all scenarii, the proposed fully adaptive step-size algorithm achieves a considerable performance gain

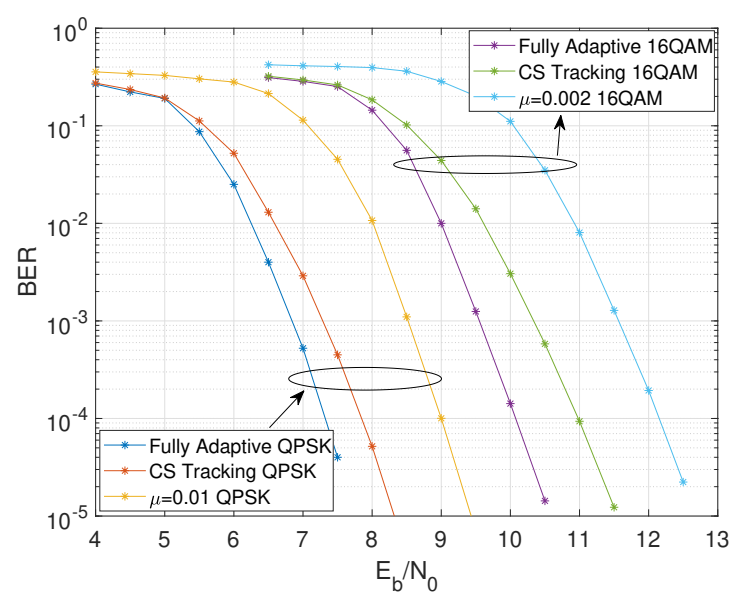

Fig. 4. BER comparison over the channel (10) for $\epsilon_{\max }^{1}=0.002$ for both the QPSK and the 16-QAM constellations.

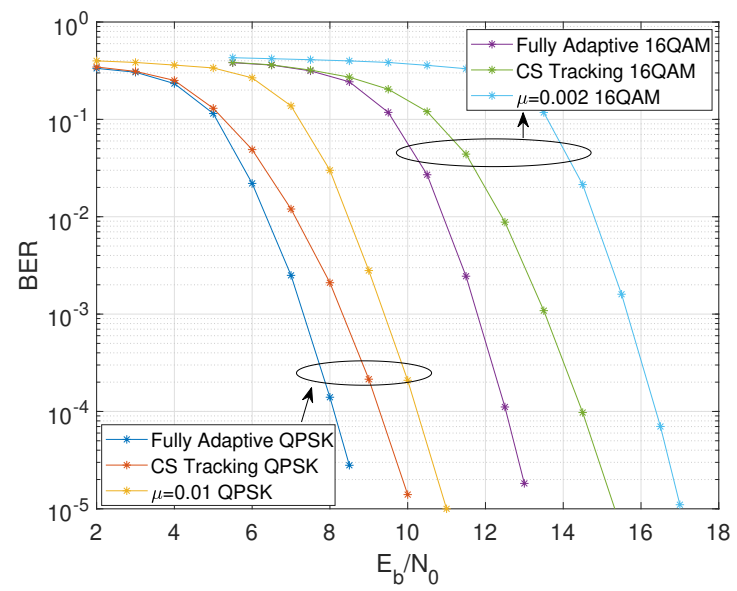

Fig. 5. BER comparison over the channel (10) for $\epsilon_{\max }^{2}=0.005$ for both the QPSK and the 16-QAM constellations.

compared to the conventional constant step-size algorithm. Moreover, it can be observed that the gain obtained with the proposed algorithm compared to the conventional algorithm increases with the Doppler shift variation speed.

Fig. 6 displays the MSE curves for $\epsilon_{\max }^{2}=0.005$ for QPSK constellation at $8.5 \mathrm{~dB}$ for all studied algorithms. It can be observed that the proposed fully adaptive stepsize algorithm has the best tracking performance among the considered algorithms.

\section{B. Watermark [28]}

User frames of 1024 bits are coded with a turbo code of rate $1 / 3$ with generator polynomials equal to $(5,7)_{\text {octal }}$; the coded bits are then interleaved and transformed into a sequence of M-QAM symbols. A training sequence of 511 symbols is transmitted before each user frame and $p_{\max }$ is fixed to 5 . The information is transmitted at a bit rate of $1.4 \mathrm{kbit} / \mathrm{s}$ at a $35 \mathrm{kHz}$ carrier frequency over the BCH1 channel [28] (e.g. 


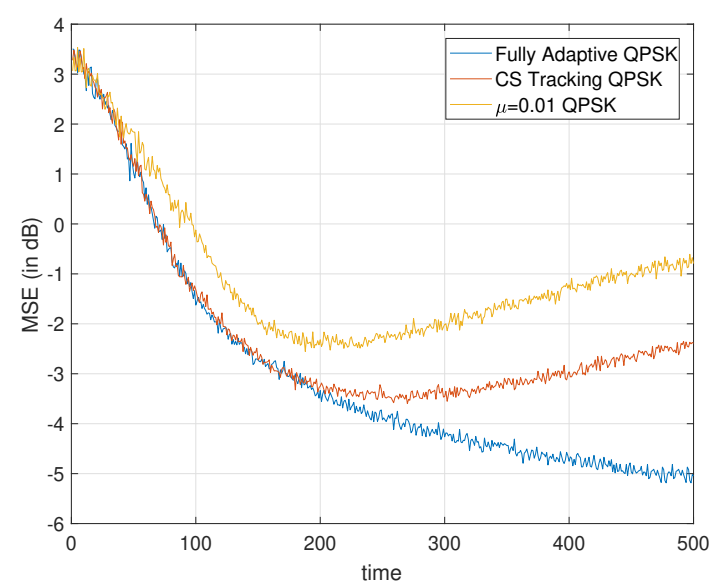

Fig. 6. MSE comparison for $\epsilon_{\max }^{2}=0.005$ for the QPSK constellation

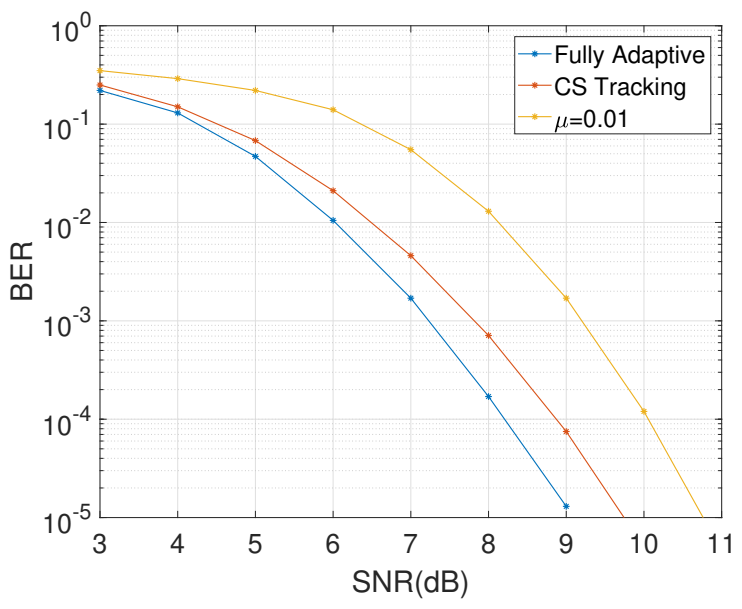

Fig. 7. BER comparison over the channel BCH1 [28].

Fig. 2). The number of taps for $\boldsymbol{f}_{k}$ and $\boldsymbol{g}_{k}$ are 21 and 31 respectively.

Fig. 7 compares the BER performance between the proposed fully adaptive step-size algorithm, the so-called "CS tracking" algorithm for which the step-size is adaptively updated over the preamble, followed by a tracking period with a constant step-size obtained at the end of the preamble, and finally the algorithm for which the step size is fixed to the initial value for all symbols transmitted over the underwater channel $\mathrm{BCH} 1$ for the QPSK constellation. It can be observed that the proposed fully adaptive step-size algorithm achieves a gain of 1.5 to 2 $\mathrm{dB}$ compared to the conventional constant step-size algorithm.

\section{CONCLUSION}

This paper proposes an original new joint equalizer - phase estimator algorithm with adaptive step-sizes. It leads to good BER performance by achieving a good trade-off between the convergence speed and accuracy over time-varying channels. Simulation results of our proposal shows a significant BER improvement compared to the conventional solutions, in particular for time-varying channel with high Doppler shifts. Finally, in the future it seems very interesting to design fully adaptive algorithms for equalization and phase synchronisation for rotated constellations [29]-[34] so as to take advantage of the inherent diversity of rotated constellations without any bandwidth loss.

\section{REFERENCES}

[1] C. Douillard, M. Jezequel, C. Berrou, A. Picart, and P. Didier, "Iterative correction of intersymbol interference: turbo-equalization," European Transactions on Telecommunications, 6 (5), pp. 507-512, Sep 1995.

[2] C. Laot, A. Glavieux, and J. Labat, "Turbo equalization: adaptive equalization and channel decoding jointly optimized,' IEEE Journal on Selected Areas in Communications, 19, pp. 1744-1752, Sep 2001.

[3] M. Tuchler and A. C. Singer, "Turbo equalization: An overview," IEEE Transactions on Information Theory, 57(2), pp. 920-952, Feb 2011.

[4] J. Li, Y. V. Zakharov, and B. Henson, "Multibranch autocorrelation method for doppler estimation in underwater acoustic channels," IEEE Journal of Oceanic Engineering, 43(4), pp. 1099-1113, Oct 2018.

[5] S. Kaddouri, P. J. Beaujean, P. Bouvet, and G. Real, "Least square and trended doppler estimation in fading channel for high-frequency underwater acoustic communications," IEEE Journal of Oceanic Engineering, 39(1), pp. 179-188, Jan 2014.

[6] J. Yang and B. Geller, "Near Optimum Low Complexity Smoothing Loops for Dynamical Phase Estimation-Application to BPSK Modulated Signals," IEEE Transactions on Signal Processing, vol. 57, no. 9, pp. 3704-3711, Sept. 2009, doi: 10.1109/TSP.2009.2021452.

[7] Z. Ye, T. Arbi, and B. Geller, "Low complexity adaptive turbo equalization for multipath underwater communication,' In OCEANS 2019 Marseille, pages 1-5, Jun. 2019.

[8] B. Geller, V. Capellano, J.-M. Brossier, A. Essebbar and G. Jourdain, "Equalizer for video rate transmission in multipath underwater communications," IEEE Journal of Oceanic Engineering, vol. 21, no. 2, pp. 150-155, April 1996, doi: 10.1109/48.486790.

[9] B. Geller, V. Capellano and G. Jourdain, ’Equalizer for real time high rate transmission in underwater communications," 1995 International Conference on Acoustics, Speech, and Signal Processing, 1995, pp. 3179-3182 vol.5, doi: 10.1109/ICASSP.1995.479560.

[10] B. Geller, J.M. Broissier, and V. Capellano, "Equalizer for high data rate transmission in underwater communications," Proc. of OCEANS IEEE Oceanic engineering, pp. 302-306, Brest, Sept. 1994.

[11] B. Benammar, N. Thomas, C. Poulliat, M. Boucheret, and M. Dervin, "On linear mmse based turbo-equalization of nonlinear volterra channels," IEEE ICASSP, pages 4703-4707, May 2013.

[12] J. W. Choi, T. J. Riedl, K. Kim, A. C. Singer, and J. C. Preisig, ”Adaptive linear turbo equalization over doubly selective channels," IEEE Journal of Oceanic Engineering, 36(4), pp. 473-489, Oct 2011.

[13] H. Lou and C. Xiao, "Soft-decision feedback turbo equalization for multilevel modulations," IEEE Transactions on Signal Processing, 59(1), pp. 186-195, Jan 2011.

[14] J.M. Brossier, P.O. Amblard, B. Geller, "Self-adaptive PLL for general QAM constellations," 2002 11th European Signal Processing Conference, Proc. EUSIPCO, pp. 631-635, Sept. 2002.

[15] A. Ozen, Ismail Kaya, and B. Soysal, "Variable step-size constant modulus algorithm employing fuzzy logic controller,' Wireless Personal Communications, 54, pp. 237-250, Jul 2010.

[16] Hong Woo, "Variable step size lms algorithm using squared error and autocorrelation of error. Procedia Engineering," 41, pp. 47-52, Dec. 2012.

[17] R. Gerzaguet, L. Ros, F. Belveze, and J.-M. Brossier, ”On multiplicative update with forgetting factor adaptive step size for least mean-square algorithms," 25th International Conference on Telecommunications (ICT), pages 588-592, June 2018.

[18] M. Gong, F. Chen, H. Yu, Z. Lu, and L. Hu, "Normalized adaptive channel equalizer based on minimal symbol-error-rate," IEEE Transactions on Communications, 61(4), pp. 1374-1383, April 2013.

[19] X. Zhong, F. Chen, F. Ji, and H. Yu, "Variable step-size least-symbol error-rate adaptive decision feedback turbo equalization for underwater channel," OCEANS 2016 MTS/IEEE Monterey, pages 1-4, Sep 2016. 
[20] S. Chen, S. Tan, Lei Xu, and L. Hanzo, "Adaptive minimum error-rate filtering design: A review," Signal Processing, 88, pp. 1671-1697, July 2008

[21] C. Berrou, A. Glavieux and P. Thitimajshima, "Near Shannon limit errorcorrecting coding and decoding: Turbo-codes," Proceedings of ICC '93 - IEEE International Conference on Communications, 1993, pp. 1064 1070 vol.2, doi: 10.1109/ICC.1993.397441.

[22] B. Geller, I. Diatta, J. P. Barbot, C. Vanstraceele and F. Rambeau, ’Block Turbo Codes: From Architecture to Application," IEEE International Symposium on Information Theory, ISIT 2006, pp. 1813-1816, July 2006.

[23] I. Nasr, B. Geller, L. Najjar Atallah, S. Cherif, "Performance Study of a Near Maximum Likelihood Code-Aided Timing Recovery Technique,' IEEE Transactions on Signal Processing, pp. 799-811 64(3), Feb. 2016.

[24] I. Nasr, L. N. Atallah, S. Cherif and B. Geller, "Near MAP Dynamical Delay Estimator and Bayesian CRB for Coded QAM Signals," IEEE Transactions on Wireless Communications, vol. 17, no. 1, pp. 636-651, Jan. 2018, doi: 10.1109/TWC.2017.2769043.

[25] Imen Nasr, Leila Najjar Atallah, Sofiane Cherif, Benoit Geller, and Jianxiao Yang, "A soft maximum likelihood technique for time delay recovery," In Fourth International Conference on Communications and Networking, ComNet-2014, pages 1-5, 2014

[26] J. Yang, B. Geller and T. Arbi, "Proposal of a multi-standard transceiver for the WBAN Internet of Things," 2016 International Symposium on Signal, Image, Video and Communications (ISIVC), pp. 369-373, 2016, doi: 10.1109/ISIVC.2016.7894017.

[27] T. Arbi, J. Yang, B. Geller, "Récepteur multi-normes pour les réseaux de capteurs de l'iot médical,' Internet des objets, 1 (Numéro 1), 2017.

[28] P. A. van Walree, F. X. Socheleau, R. Otnes, and T. Jenserud, "The "watermark" benchmark for underwater acoustic modulation schemes," IEEE Journal of Oceanic Engineering, 42(4), pp. 1007-1018, Oct. 2017.

[29] T. Arbi, B. Geller, J. Yang, C. Abdel Nour and O. Rioul, "Uniformly Projected RCQD QAM: A Low-Complexity Signal Space Diversity Solution Over Fading Channels With or Without Erasures," IEEE Transactions on Broadcasting, vol. 64, no. 4, pp. 803-815, Dec. 2018, doi: 10.1109/TBC.2018.2811618.

[30] T. Arbi and B. Geller, "Joint BER Optimization and Blind PAPR Reduction of OFDM Systems With Signal Space Diversity," IEEE Communications Letters, vol. 23, no. 10, pp. 1866-1870, Oct. 2019, doi: 10.1109/LCOMM.2019.2931898.

[31] T. Arbi, I. Nasr and B. Geller, "Near Capacity RCQD Constellations for PAPR Reduction of OFDM Systems," ICASSP 2020 - 2020 IEEE International Conference on Acoustics, Speech and Signal Processing (ICASSP), pp. 5110-5114, 2020, doi: 10.1109/ICASSP40776.2020.9053923.

[32] T. Arbi, Z. Ye and B. Geller, "Low-Complexity Blind PAPR Reduction for OFDM Systems With Rotated Constellations," IEEE Transactions on Broadcasting, vol. 67, no. 2, pp. 491-499, June 2021, doi: 10.1109/TBC. 2021.3056232.

[33] J. Yang, K. Wan, B. Geller, C. Abdel Nour, O. Rioul and C. Douillard, "A low-complexity 2D signal space diversity solution for future broadcasting systems," 2015 IEEE International Conference on Communications (ICC), pp. 2762-2767, 2015, doi: 10.1109/ICC.2015.7248744.

[34] Jianxiao Yang, Meng Li, Min Li, Charbel Abdel Nour, Catherine Douillard, and Benoit Geller, "Max-log demapper architecture design for DVB-T2 rotated QAM constellations," IEEE Workshop on Signal Processing Systems (SiPS), pages 1-6, Dec. 2015. 